\title{
Estado, economía y política: las intervenciones sociales del estado orientadas al mercado de trabajo en la provincia de Córdoba entre 1983 y 1995
}

[State, Economy and Politics: the Social Interventions of the State in the Labor Market in the Province of Córdoba between 1983 and 1995]

\author{
Julieta A. Almada \\ (CEA/CONICET - Universidad Nacional de Córdoba) \\ julialmada@gmail.com
}

\begin{abstract}
Resumen:
El presente trabajo analiza las intervenciones sociales del estado en materia de regulación del mercado de trabajo en Córdoba entre 1983 y 1995 impulsadas por el Poder Ejecutivo y el Poder Legislativo. En nuestro abordaje analizamos si estas pueden ser consideradas, o no, como complementarias a las impulsadas en el ámbito nacional en lo que respecta a la re-regulación de las relaciones laborales en el marco de los nuevos requerimientos del régimen social de acumulación por valorización financiera.
\end{abstract}

Palabras clave: Mercado de trabajo Provincia de Córdoba - Intervenciones sociales del estado

\begin{abstract}
:
This paper analyzes the social interventions of the state in the regulation of the labor market in Córdoba between 1983 and 1995, promoted by the Executive Power and the Legislative Power. In our approach, we analyze whether or not these can be considered complementary to those promoted at the national level with respect to the re-regulation of labor relations within the framework of the new requirements of the social regime of accumulation by financial valorization.
\end{abstract}

Keywords: Labor market - Province of Córdoba - State social interventions

Recibido: $10 / 5 / 2018$

Evaluación: 24/8/2018

Aceptado: 21/2/2019

Anuario de la Escuela de Historia Virtual - Año 10 - N 15 - 2019: pp. 68-90. ISSN: 1853-7049

http://revistas.unc.edu.ar/index.php/anuariohistoria 


\section{Estado, economía y política: las intervenciones sociales del estado orientadas al mercado de trabajo en la provincia de Córdoba entre 1983 y 1995}

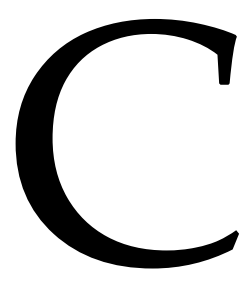

on la apertura democrática el radicalismo triunfó en la provincia de Córdoba de la mano de Eduardo César Angeloz, quien gobernó ininterrumpidamente hasta 1995. El predominio provincial del partido radical marcó, según la bibliografía existente, un proceso distintivo en relación con el ámbito nacional, que fue haciéndose más evidente y relevante en función del cambio del partido gobernante. Luego del triunfo de Carlos Menem, que rezagó a un segundo lugar al gobernador de Córdoba en la disputa por la presidencia de la nación, Angeloz impulsó la Ley Provincial 7.850 de Reforma Administrativa y Económica, un plan de "modernización del aparato estatal" que posibilitaba el traspaso de organismos y empresas dependientes del Estado provincial a manos privadas (Closa, 2010). Así, diversos autores afirman que, si bien los objetivos de la reforma provincial estaban, en términos generales, en consonancia con las transformaciones estatales a nivel nacional (disminución del gasto público, incorporación de capital privado y descentralización administrativa), esta adquirió rasgos particulares que posibilitarían pensar en un desfasaje temporal (Gordillo, 2003). Por otra parte, el análisis de este proceso ha permitido a otros autores (La Serna, 2001; Carrizo, 1997; Gordillo, 2003) caracterizar a los gobiernos angelocistas como un caso de Estado provincial con rasgos bienestaristas. Esta interpretación se sostiene sobre el análisis de los derechos de inclusión ciudadana en la prestación de servicios sociales, cuya cobertura sería de corte universalista -tanto en materia de salud como de educación- combinado con la presencia de servicios asistenciales de baja institucionalidad y alta discrecionalidad.

Otro rasgo que se destaca en la producción académica local sobre el período es que, frente a la reforma estatal a nivel nacional que posibilitaba el creciente fortalecimiento del poder ejecutivo, los mecanismos de legitimación política en Córdoba se caracterizaron por una forma neo-corporativa (La Serna, 1997). A partir de estos elementos, se ha propuesto caracterizar al modelo cordobés dentro de una matriz estadocéntrica (Gordillo, 2003), que le otorga al Estado el papel principal de organizador y mediador de lo social a partir de mecanismos de concertación y acuerdo con los diferentes actores sociales.

Es preciso reconocer que los análisis locales que aportaron a la caracterización de este proceso histórico abordaron escasamente los asuntos socio-económicos, en los que pretendemos enfocarnos en este trabajo. En particular, son fragmentarias las referencias 
respecto de las intervenciones sociales del estado con relevancia en la regulación del mercado de trabajo o, incluso, al abordaje de la estructura económica provincial.

En esta investigación entendemos que el Estado, a través de sus intervenciones sociales, regula -entre otros aspectos- el mercado de trabajo, por lo menos, "a través de tres instrumentos: las medidas de política laboral (que incluyen la legislación del trabajo y las políticas hacia el mercado de trabajo), la provisión estatal de bienes, servicios y transferencias y la seguridad social" (Cortés y Marshall, 1991, p. 22). De manera precisa, la categoría de intervención social del estado pretende ampliar la restrictiva idea de la política social como provisión gratuita de bienes y servicios, tales como educación, salud y vivienda.

La noción de intervención social del estado incluye tanto los instrumentos de regulación en el área laboral como también las políticas poblacionales, demográficas y hacia la familia que tiene también efectos, aunque quizás menos deliberados al momento de su concepción, sobre el mercado de trabajo. Del conjunto de instrumentos o mecanismos de la intervención social del estado, en este trabajo nos centraremos en la legislación laboral -el derecho individual y colectivo de trabajo dirigido a regular deliberadamente el mercado de trabajo y el conflicto social- y las denominadas estrictamente "políticas hacia el mercado de trabajo" tales como, políticas de empleo como por ejemplo las dirigidas a la empleabilidad de ciertos grupos- (Cortés y Marshall, 1993). Nos concentraremos en la legislación del trabajo en tanto "es uno de los instrumentos cruciales de que dispone el Estado para regular el mercado de trabajo y el conflicto, siguiendo los lineamientos de la política laboral" (Marshall, 2000, pp. 452-453). En este sentido, la legislación del trabajo -entendida en un sentido amplio que incluye las normas de carácter legal que no son propiamente leyes-, constituye un instrumento de regulación capaz de impulsar cambios en el mercado de trabajo y en la forma en que se procesan los conflictos laborales. Por último, quisiéramos destacar que la legislación laboral es asumida como resultado de compromisos políticos y sociales que reflejan las posiciones de diferentes expresiones políticas e intereses sectoriales y corporativos y de los diversos ámbitos institucionales del complejo estatal. Según Marshall (1996), los proyectos legislativos representan una de las expresiones más claras de la ideología y la política laboral del Estado.

A partir de tales consideraciones, nuestro problema procura establecer cómo se articularon las intervenciones sociales del estado durante el gobierno de Eduardo Angeloz con la configuración del mercado de trabajo local. Pondremos nuestros esfuerzos en presentar las principales intervenciones sociales del estado, impulsadas por el Poder Ejecutivo y por el Poder Legislativo de la Provincia de Córdoba comparando su orientación general con las promovidas desde el ámbito nacional. Considerando que estas últimas estuvieron orientadas a promover una re-configuración del mercado de 
trabajo procurando que las características y volumen de la oferta de trabajo fueran compatibles con los requerimientos del patrón de acumulación por valorización financiera vigente en aquellos años, es nuestro interés analizar y caracterizar las orientaciones de las intervenciones sociales del estado a nivel provincial. En particular, analizaremos si estas pueden ser consideradas, o no, como complementarias a las impulsadas en el ámbito nacional en lo que respecta a la re-regulación de las relaciones laborales.

\section{El régimen social de acumulación por valorización financiera y los cambios en el mercado de trabajo}

El golpe de Estado cívico-militar perpetrado el 24 de marzo de 1976 introdujo profundas transformaciones que afectaron duraderamente a la sociedad argentina, y que se profundizaron en los años '90. En el plano económico-político, la interrupción del modelo de sustitución de importaciones -que había estructurado las relaciones económicas y sociales desde mediados del siglo XX- llegaría a partir de la imposición de políticas económicas de orientación neoliberal. En particular, las principales transformaciones se promovieron con la apertura comercial de la economía y la reforma financiera destinada a liberalizar el mercado de capitales; es decir, los cimientos que provocaron un proceso general de desindustrialización, con profundas consecuencias para la transformación de la estructura económica del país, impulsando un nuevo patrón de acumulación por valorización financiera ${ }^{1}$ (Basualdo, 2010).

Las transformaciones impulsadas importaron una profunda modificación del lugar del Estado como organizador de las actividades económico-sociales. Como parte de esta importante restructuración, se produjo un proceso de concentración y centralización del capital (Ortiz y Schorr, 2006), que dio lugar a una trascendente declinación de la pequeña y mediana empresa, así como también, una significativa reconfiguración de la organización social del trabajo. En tanto en el nuevo régimen de acumulación se diluía el papel que la demanda salariada había asumido durante la vigencia del modelo de industrialización sustitutiva, los trabajadores fueron perdiendo los derechos laborales conquistados a lo largo del siglo XX.2

\footnotetext{
${ }^{1}$ Es necesario reconocer que esta perspectiva ha tenido una profusa literatura crítica, entre otros podemos citar a Sartelli, Kabat et al., (1998); Fitzsimons (2013); Pérez Álvarez (2013); Grigera (2008; 2011 y 2013). Las primeras focalizan para establecer sus explicaciones en las transformaciones internacionales que darían cuenta de una temprana mutación del sistema capitalista a nivel global. Desde nuestra perspectiva, si bien es importante considerar el desenvolvimiento global del capitalismo, resulta fundamental ponderar las dinámicas nacionales, provinciales y locales, ya que la agencia de los actores locales puede contribuir a adaptar o impulsar transformaciones que matizan y modifican esas líneas generales del proceso de acumulación global. Otras periodizaciones posibles son planteadas desde perspectivas liberales como las que podemos encontrar en Gerchunoff y Llach (1999) o Novaro y Palermo (2003 y 2004). Un estudio pormenorizado del asunto del agotamiento de la ISI puede encontrarse en Reche (2015).

2 Otros estudios periodizan de manera diferente este proceso, identificando un retroceso de derechos de los trabajadores ya desde los años '50. Véase, por ejemplo, James (1981), Marticorena (2010), Kabat, Harari et al. (2014). Por cierto, es preciso destacar que si bien la incorporación del capital trasnacional en la producción
} 
Según la perspectiva que seguimos, cambios de la magnitud de los señalados, implican necesariamente transformaciones sustantivas en la configuración del mercado de trabajo, ya que este debe adecuarse a los requerimientos de la forma de valorización de capital dominante en cada contexto socio-histórico particular (Cortés y Marshall, 1991). Con posterioridad, el efecto disciplinador de la hiperinflación y de las devaluaciones impulsadas fueron el escenario de aprobación de las Leyes de Emergencia Económica y de Reforma del Estado (Basualdo, 2010). En particular, en la nueva estrategia socio-económica, las protecciones sociales se transformaron en un obstáculo para la "modernización" de la economía argentina que debía orientar su producción a las "demandas del mercado" mundial. Por ello, la legislación laboral buscó adecuar la dinámica del mercado de trabajo al nuevo patrón de acumulación de capital, modificando las regulaciones laborales que hasta entonces protegían al trabajador (Lindenboim y González, 2004).

\section{La economía provincial y la dinámica del empleo urbano}

Resulta relevante destacar que, en el marco del proceso de industrialización por sustitución de importaciones, Córdoba había ocupado un papel destacado como productora de bienes industriales. Estas actividades generaron numerosos eslabonamientos productivos que estimularon el desarrollo económico regional a partir de su conocido papel de dinamizador (Brennan y Gordillo, 2008). Especialmente, se promovió la generación de proyectos asociados a la industria metal-mecánica, como proveedores y productores de insumos trabajo-intensivos. En este sentido, Córdoba adquirió los rasgos de un polo industrial y un centro receptor tanto de mano de obra como de materia prima durante la vigencia del régimen de acumulación basado en la sustitución de importaciones (Rofman y Romero, 1997).

La relevancia en el entramado económico y la centralidad para su propia economía explican que las políticas implementadas desde 1976 afectaran particularmente la realidad económica y social de nuestra provincia (Almada y Reche, 2019). A partir de 1978 es notoria la reducción de la participación de las industrias manufactureras en el Producto Bruto Geográfico, tendencia que se hará cada vez más pronunciada, conforme se intensifiquen las políticas liberalizadoras. Sin dudas, la apertura indiscriminada de la economía que llevó adelante la gestión económica de la dictadura cívico-militar afectó particularmente al sector automotriz cordobés (Gatto, Gutman y Yoguel, 1988). Con el proceso aperturista, las importaciones tanto de bienes terminados, como de autopartes

local se intensifica y genera nuevas pautas de organización de la producción y el trabajo, las normativas laborales, eje de este trabajo, comienzan a modificarse con una orientación crecientemente liberal con posterioridad. 
crecieron significativamente. Asimismo, aunque a partir de 1981 volvió a constituirse como una actividad económica protegida, quedó abierta la importación de partes, por lo menos, hasta $1984 .^{3}$ Esto contribuyó a que los componentes de producción nacional fueran disminuyendo cada vez más, con el consecuente cierre de talleres industriales y la destrucción de puestos de trabajo en el sector más importante para el empleo local: el de producción de autopartes. Así, en una provincia con una economía central y densamente integrada al modelo mercado-internista, las transformaciones ocurridas en el marco del nuevo régimen de acumulación generaron un particular impacto en un mercado de trabajo estructurado en función de las actividades más dinámicas del patrón de acumulación que quedaron atrás.

Según es posible observar, la mayoría de los indicadores laborales y ocupacionales dan cuenta de un peor desempeño y de situaciones de mayor gravedad en el deterioro general de las que expresan los mismos indicadores a nivel nacional. Durante el período, el constante crecimiento poblacional -por crecimiento vegetativo, sumado a los procesos migratorios- y de la PEA -además por la incorporación en la oferta de nuevos segmentos poblacionales, en particular, jóvenes, adultos mayores y mujeres-, no estuvo acompañado de un incremento de la demanda de fuerza de trabajo, que permitiera absorberla. Específicamente podemos afirmar que el ritmo de creación de empleos públicos y privados -e incluso la destrucción de puestos de trabajo- no permitió la incorporación efectiva de esa creciente oferta de fuerza de trabajo. En este marco, tomando como punto de partida el año 1983, la desocupación del Gran Córdoba se triplicó en 1995; así, si en 1983 la tasa de desocupación era del 5,59\%, hacia 1995 se había elevado al 15,94\%. Además, al igual que las lecturas nacionales, es posible advertir, para el caso provincial, que la tasa de desocupación presenta, ya en los años ochenta, una tendencia alcista.

Por otro lado, la tasa de actividad fue mayor respecto al total de aglomerados urbanos del interior del país. Su incremento, sobre todo en años de crisis, se puede explicar por la incorporación de nuevos trabajadores -en particular jóvenes, adultos mayores y mujeres- como oferta de fuerza de trabajo. Sin embargo, la tasa de empleo presentó un nivel menor a la del promedio nacional y del Gran Buenos Aires -excepto en los años 1983, 1985 y 1990-. Por su parte, la tasa de subocupación siguió la evolución común al total de aglomerados urbanos del país, aunque levemente por encima del promedio nacional, exceptuando los años 1987, 1988 y 1990 en los que se observa un porcentaje de subocupación menor respecto al del total de los aglomerados urbanos. Se pueden advertir, además, alteraciones importantes de esta tasa en breves periodos de tiempo,

\footnotetext{
${ }^{3}$ Es preciso advertir que los cambios en la estructura del sector automotriz son también resultado de modificaciones en la organización internacional de la actividad, luego de la crisis de fines de los '70 y comienzos de los '80 y de la profunda recesión en los '80 que redujo el consumo interno, principal destino por ese entonces de la producción del complejo automotriz, tal como destacan entre otros Tarditi $(1999,2000)$ y Bil (2017). Asimismo, es necesario destacar que, en cuanto a la importación autopartista, el decreto 4070/84 sujetó hasta 1988 la importación de piezas a un régimen de consulta previa con representantes de las cámaras autopartistas.
} 
que se presentan como un rasgo característico del mercado de trabajo urbano local durante la valorización financiera (Almada, Giraudo y Schuster, 2015).

Así, la subutilización de la fuerza de trabajo se evidenció como una constante de la configuración del mercado de trabajo urbano durante todos los años comprendidos en esta investigación. Desde la recuperación democrática la adición de desocupación y subocupación se mantuvo entre el 13 y 14\% aproximadamente y desde 1991 indicó un promedio de 19\%. En la crisis de 1995 alcanzó al 29,7\% de la población económicamente activa, elemento que da cuenta de un deterioro del mercado de trabajo cordobés y una creciente vulnerabilidad laboral.

\section{Las vicisitudes jurisdiccionales de las intervenciones sociales del estado sobre el trabajo}

En el período bajo estudio se producen numerosas transformaciones no solo en materia de legislación laboral, sino también en las incumbencias estatales en las diferentes jurisdicciones. Si bien existen algunos estudios que detallan con minuciosidad las primeras (Neffa, 1998; Marshall, 1996; 2000; Gaudio y Tomada, 1991; Zorzoli, 2015; Meik y Zaz, 1990; Fernández, 2005; Palomino 2005; Peñalva, 2001) no existe, hasta el momento, un relevamiento de las atribuciones provinciales en materia de regulación laboral que den cuenta no solo del proceso de creciente descentralización administrativa, sino también y co-constitutivamente, de ampliación de las capacidades estatales provinciales para regular ya no solo el empleo público local, sino también el privado. En este sentido, destacar los cambios en las incumbencias nacionales y provinciales en materia de regulación laboral, resulta fundamental para comprender luego las re-regulaciones impulsadas desde el ámbito provincial, en la medida en que las provincias se constituyen como espacios de producción de lo político y social, articuladas con la escala nacional, pero respecto de la cual conservan una autonomía relativa, que es imprescindible recuperar (Servetto y Moyano, 2009).

Antes de detenernos en analizar la labor desarrollada por el Ministerio de Trabajo Provincial, ${ }^{4}$ resulta importante presentar la distribución de funciones entre nación y provincia en el ámbito laboral, tal como quedaron establecidas a partir del convenio de 1986 y con los posteriores acuerdos de 1990.

El 31 de marzo de 1986 se firmó un nuevo convenio en el que se estableció una división de funciones entre las jurisdicciones sobre el mercado de trabajo (público y privado), dejando atrás el acta acuerdo de 1974 entre Nación y el gobierno interventor de la Provincia de Córdoba. En aquel se delimitó las funciones en el ámbito laboral provincial y fue ratificado por el poder legislativo provincial que lo convirtió en la Ley

\footnotetext{
${ }^{4}$ El Ministerio de Trabajo en la Provincia de Córdoba se crea como Secretaría-Ministerio en 1985, desde entonces y hasta 1995, a la cabeza del abogado laboralista, miembro de la UCR, Jorge Sappia.
} 
№ 7.478 en diciembre del mismo año. Por medio de este acto se estableció que la Nación se reservaba la competencia para regular la política salarial, las negociaciones colectivas, la homologación y la aplicación e interpretación de las convenciones colectivas de trabajo del empleo privado y público nacional. Además, quedaría bajo su órbita el régimen de asociaciones profesionales, así como los conflictos colectivos que tuvieran lugar en organismos o empresas del Estado Nacional o cualquier otra circunstancia que afectara o comprometiera el interés nacional. De igual forma se reservaba la potestad para la calificación de insalubridad dentro del ámbito laboral.

Por su parte, la provincia se comprometió a ejercer el poder de policía en todo el ámbito de la provincia, tanto público como privado, y a intervenir en los conflictos colectivos e individuales suscitados dentro del ámbito público provincial. También se le atribuía la fiscalización de la Ley de Higiene y Seguridad (19.587) y se comprometía a la organización de un servicio de empleo privado que luego sería coordinado por el Sistema Nacional de Empleo.

Más adelante, y con un nuevo signo político en el gobierno nacional, el 17 de diciembre de 1990 se celebró un nuevo acuerdo por medio del cual se le reconoció a la provincia de Córdoba mayores facultades en materia del ejercicio de poder de policía laboral. En el nuevo convenio se expresaba que su finalidad era afianzar y promover el funcionamiento autónomo de las administraciones provinciales en el ejercicio pleno del poder de policía del trabajo. Además de las funciones que ya estaban convenidas en el acuerdo anterior se estableció que el Gobierno Provincial tendría, a partir de ese momento, las atribuciones de: a) efectuar la determinación y calificación de ambientes insalubres; b) realizar la gestión administrativa completa de los infortunios laborales; c) aplicar y gestionar las Convenciones Colectivas de Trabajo y; d) aplicar y gestionar la negociación colectiva con sus agentes públicos.

Según la concepción de la iniciativa, la concesión de autoridad al Ministerio de Trabajo Provincial en la intervención de las negociaciones colectivas del ámbito privado tenía como objetivo "la descentralización en esta materia, privilegiando la participación de los actores sociales locales, lo que redundará en la implementación de un marco normativo acorde a las características de la economía regional". ${ }^{5}$ En 1991, a través de la ley provincial 8.072, se ratificó el convenio firmado entre la Nación y la Provincia y, por medio de esta quedó sancionada la potestad de intervención en las negociaciones colectivas también en el ámbito privado provincial.

\section{Intervenciones sociales del estado y sus orientaciones en clave provincial}

A lo largo del período podemos trazar ciertas líneas de intervención fundamentales que nos permitirán dar cuenta de las orientaciones del gobierno provincial hacia la regulación de las relaciones laborales y, al mismo tiempo, esbozar una subperiodización

\footnotetext{
${ }^{5}$ Biblioteca de la Honorable Legislatura de la Provincia de Córdoba (en adelante, BHLC), Informe de la Secretaría-Ministerio de Trabajo (en adelante IMT), 1991, p. 157.
} 
de nuestro objeto de estudio. En este sentido, podemos identificar regularidades en dos grandes núcleos temporales; el primero (de 1983-1986), donde el objetivo primordial es la "normalización institucional"; el segundo (de 1987-1995), cuya orientación es, justamente, la re-regulación de las relaciones laborales, posible a partir de la nueva normativa descentralizadora que estableció mayores facultades de intervención en materia de regulación laboral a la provincia. Una sub-división específica, además, la realizamos a partir de 1991, con la nueva Ley Nacional de Empleo, cuando comienza un proceso de readecuación a tal normativa. Asimismo, en estos últimos años, se profundizan dos líneas de intervención en las políticas de empleo que habían sido ya esbozadas con anterioridad: la "colocación" de trabajadores y los diversos programas de capacitación para la reconversión productiva.

\section{Normalización institucional}

La etapa entre 1983 y 1986 podríamos denominarla de normalización institucional ya que, tanto desde el Ejecutivo con la creación de la Secretaría-ministerio de trabajo, como desde el poder legislativo provincial, comienza un proceso de rehabilitación institucional de delegaciones y subdelegaciones del Ministerio de Trabajo (nacional) que habían sido cerradas durante la última dictadura cívico-militar. En estos primeros años, las principales intervenciones relevadas refieren, entonces, a la reinstalación de las subdelegaciones del Ministerio de Trabajo de la Nación en las cabeceras departamentales de la provincia, así como la creación de nuevas delegaciones. ${ }^{6}$

En este contexto, dentro de los argumentos presentados ante la Honorable Cámara legislativa, se justificaba la restitución del personal de las subdelegaciones e inspectorías -en acuerdo tanto del bloque radical como del justicialista- en tanto se planteaba que era necesario reparar el daño infringido por la última dictadura militar que, a partir del cierre de las subdelegaciones del interior y el cesanteo de su personal, provocó "el retorno al autoritarismo patronal y a la desprotección del obrero". Así, mediante el restablecimiento de las subdelegaciones se aseguraba "a los trabajadores que la tutela de sus derechos no se transforme en simples actitudes declarativas, sino que se efectivicen en la práctica".

Por otro lado, la normalización institucional, puede ser analizada también en los crecientes reclamos por la restitución de trabajadores cesanteados en tiempos

\footnotetext{
${ }^{6}$ A modo de ejemplo, en 1984 se reinstalaron las subdelegaciones del Ministerio de Trabajo de la Nación en La Carlota, Deán Funes, Cruz del Eje, Villa María, San Francisco, Jesús María, Salsacate, Alta Gracia, Villa Dolores, Bell Ville, Marcos Juárez, Río Cuarto, Laboulaye y en las demás cabeceras departamentales; asimismo, se crearon dos nuevas subdelegaciones en Río III y Morteros.

${ }^{7}$ BHLC, Diario de Sesiones de la Honorable Cámara de Diputados de la provincia de Córdoba (en adelante DSD), 1984 (12/6), pp. 269-271.
} 
dictatoriales. En este sentido, la reinstalación de subdelegaciones y creación de nuevas significó no solo una ampliación en términos del personal comprometido en ejercer la función de policía laboral en las localidades del interior provincial, elemento que se verá representado también en las cantidades de inspecciones realizadas, ${ }^{8}$ tanto en la ciudad capital como en localidades del interior, sino también la restitución de los trabajadores cesanteados en estos organismos durante la última dictadura cívico-militar. Según se puede extraer de las argumentaciones presentadas en los reiterados tratamientos y referencias al tema, se entendía que las medidas adoptadas por el gobierno de facto habían atentado contra los derechos y garantías establecidas en el artículo 14 bis de la Constitución Nacional, en lo referido a la estabilidad del empleado público. ${ }^{9}$ Cabe destacar que los proyectos para reincorporar a trabajadores cesanteados, por causas políticas o gremiales, a partir del 24 de marzo de 1976, no se limitaron exclusivamente al ámbito del Ministerio de Trabajo y las subdelegaciones provinciales; muy por el contrario, involucraba a trabajadores del ámbito público y privado.

\section{Reorientación de las intervenciones sobre el trabajo.}

Reconversión de la mano de obra y eficiencia en la reforma del Estado provincial

Los idearios de transformación para la "modernización", propuestos por el poder ejecutivo provincial, cristalizaron en la reforma de la constitución provincial. Con el apoyo del peronismo, se llevó adelante la modificación de la carta magna, que tuvo como resultados, entre otros, la incorporación de nuevas fuerzas al ámbito de la legislatura provincial, una reorientación de las funciones del Estado -en tanto se comprometía a privatizar diversas áreas y empresas estatales- y la denominada "descentralización administrativa" hacia los municipios, así como la incorporación de "nuevas instancias de participación ciudadana" como el referéndum y las consultas populares.

En la nueva constitución, se intentaban consolidar las funciones de la Secretaríaministerio de trabajo fortaleciendo su antiguo rol de mediador entre trabajo y capital, al mismo tiempo que adquiría nuevas significaciones estableciendo que "los empresarios, los trabajadores y el Estado son responsables de la eficiencia, productividad y progreso de las organizaciones económicas que participan en el proceso productivo".${ }^{10} \mathrm{El}$ discurso de la eficiencia y la productividad, que aparece en diversos sentidos en la nueva constitución provincial, puede identificarse a partir de este momento, como un elemento central de la programática económico-política de la gestión angelocista.

En su mensaje hacia la Honorable Asamblea legislativa al año siguiente de haber reformado la Constitución provincial, el riotercerense expresaba que “eficiencia, esta

\footnotetext{
8 Lamentablemente, la información estadística que contamos del accionar de la Secretaría-ministerio respecto a las inspecciones y resolución de conflictos son solo para los años 1988 y del periodo 1990-1995. ${ }^{9}$ BHLC, DSD, 1984 (5/6), p. 151.

${ }^{10}$ BO, Constitución de la Provincia de Córdoba, 1987, Art. 67.
} 
será, honorable asamblea, nuestra meta, nuestro horizonte, también nuestra obsesión". ${ }^{11}$ La reforma política emprendida sería el piso necesario para "reordenar" la economía; y en este nuevo contexto, también el Estado dejará de cumplir un rol de árbitro, productor y empresario, y limitará sus funciones a "garantizar la competitividad" y el desenvolvimiento libre de las fuerzas de mercado para lograr dirigir la producción local hacia el mercado externo. ${ }^{12}$ Asimismo, la llamada "modernización" significaba, en términos económicos y sociales, una apertura comercial que se correspondía con una estrategia económica en la que el salario dejaba de jerarquizarse según su función en la demanda para pasar a convertirse en una preocupación respecto de los costos de producción. Sobre la base de lo planteado hasta aquí, conviene preguntarse cuáles fueron los principales programas y acciones, intervenciones sociales del estado, impulsadas por el gobierno en torno al mundo del trabajo.

En primer lugar, parece importante destacar los incipientes programas de reconversión de la mano de obra que se potenciarán en estos años y que intentaban modificar las características de los trabajadores para su contratación. En lo que podríamos calificar como políticas de empleo en sentido restringido y pasivas encontramos a los Programas de Formación para Trabajadores Calificados, así como pasantías para estudiantes universitarios de la Facultad de Derecho en el ámbito del Estado y Programas de Capacitación Laboral para la Reconversión de la Mano de Obra. Los mencionados programas, dependían del Ministerio de Trabajo de la Provincia y colocaban el eje de su intervención en la empleabilidad de los sujetos ${ }^{13}$ a partir de la dotación, por parte del Estado, de ciertas capacidades/saberes de los que carecerían. Las denominadas por el Ministerio de Trabajo "políticas de empleo" tuvieron, en estos primeros años, las características de ser focalizadas y destinadas a aquellos grupos considerados por el aparato estatal como "inempleables".

Por otra parte, el gobierno angelocista, por medio del Ministerio de Trabajo, y como parte de las políticas de empleo implementadas, institucionalizó nuevos Consejos integrados por empresarios, trabajadores y el Estado para acordar las condiciones de seguridad e higiene en el ámbito laboral privado. Así se institucionalizó una acción

\footnotetext{
${ }^{11}$ BHLC, Mensaje del gobernador de la provincia de Córdoba a la Honorable Asamblea Legislativa (en adelante MG), 1988, p. 4.

12 BHLC, MG, 1988, p. 13.

${ }^{13}$ El concepto de empleabilidad sitúa la responsabilidad de su condición de desempleado en el sujeto y las causales de la situación se centran en su falta de competencias o experiencia, en el déficit educativo, etc., dejándose de lado las condiciones estructurales del mercado de trabajo marcadas por los procesos de acumulación y la dinámica del capital, que exceden la voluntad y situación individual de cada trabajador desempleado (Pérez, 2005).
} 
estatal de promoción y mediación de intereses en cuanto a las condiciones de trabajo que luego se incorporaron en los convenios colectivos de trabajo.

En el ámbito legislativo, la UDC -Unión Demócrata de Centro- contribuyó a la modificación de la agenda de discusión política de los temas a los que estamos refiriéndonos. En materia laboral, fue esta fuerza política la que tuvo un rol protagónico en la presentación de propuestas de reforma tendientes a la liberalización y desregulación del mercado de trabajo urbano privado. ${ }^{14}$ El reconocer que no hubo una impugnación explícita a tales iniciativas por parte de los partidos mayoritarios (UCR y PJ), puede ser indicador de cierto consenso en relación a un diagnóstico de necesidad de reformas laborales con orientación neoliberal. ${ }^{15}$ Asimismo, es necesario destacar que la UDC no tuvo exclusividad al elevar propuestas de modificación de las regulaciones sobre el trabajo. Tanto desde la Democracia Cristiana como desde el Partido Justicialista se realizaron presentaciones de proyectos tendientes a flexibilizar las relaciones laborales. La Democracia Cristina impulsó, ya en 1988, una propuesta para autorizar contratos a plazo fijo ${ }^{16} \mathrm{y}$ otra destinada a la incorporación de un sistema de becas dentro de la estructura productiva provincial que garantizara a las empresas privadas una "reducción de los costos de producción". ${ }^{17}$

Por otra parte, en lo que respecta al accionar del Ministerio de Trabajo, se experimentó un proceso de extensiones institucionales a partir de la creación de nuevas delegaciones en el interior provincial. Algunos años después aparecerá un nuevo tipo de justificación para la creación de subdelegaciones ministeriales: estas resultaban de interés como parte del proceso de descentralización administrativa que había encarado el gobierno de Angeloz. ${ }^{18}$ Asimismo, en estos años comenzaron a modificarse sustancialmente las normas regulatorias del ámbito laboral, así como de la justicia del trabajo. Sin dudas la modificación más importante en este sentido fue la del Código Procesal del Trabajo. ${ }^{19}$

\footnotetext{
${ }_{14}$ Aunque no todas estas propuestas fueron tratadas en la Legislatura provincial y por tanto no se convirtieron en efectivas políticas regulatorias, los posicionamientos de las principales fuerzas políticas y sociales hacia finales de este subperíodo fueron en consonancia con las nuevas propuestas impulsadas por los legisladores de la UDC.

${ }^{15}$ Un elemento que debe tenerse en cuenta para presentar algunas hipótesis explicativas de tal consenso es la permeabilidad e inserción molecular en los diferentes poderes del Estado y en los partidos políticos mayoritarios de miembros del IERAL como cuadros técnicos y asesores políticos (Fantín y Schuster 2013, p. 87).

${ }^{16}$ La propuesta fue presentada por los diputados Fernández y Manzur, ambos de Democracia Cristiana. BHLC, DSD, 1988 (9/8), p. 1847.

${ }^{17}$ En este sentido, se puede entender la propuesta de Implementación de Sistema de Becas para la Industria Provincial. BHLC, DSD, 1988 (27/9), p. 2379. Con objetivos similares, en otra ocasión el diputado peronista Alonso presentó un proyecto solicitando al PEN la suspensión de la Ley 23.696 de protección del empleo y la situación laboral. BHLC, DSD, 1990 (27/3), p. 261.

${ }^{18}$ BHLC, IMT, 1992, p. 5.

${ }^{19}$ BHLC, DSD, 1990, (13/11), p. 3855-3877.
} 
Sintonía entre Nación y Provincia en la restructuración laboral

Los primeros años de la gestión menemista estuvieron en consonancia con las propuestas de la campaña de su contrincante Eduardo Angeloz. Si Menem representaba, en su campaña de 1989, un discurso que recuperaba las banderas históricas del peronismo, proponiendo el "salariazo" y la "revolución productiva"; por el contrario, el cordobés mantendría una posición bajo los lineamientos neoliberales, resaltando los logros obtenidos en la provincia desde la recuperación democrática con un proyecto de "austeridad" económica, compatible con los requerimientos de los organismos multilaterales de crédito. Es comprensible entonces que, frente al desarrollo de las primeras medidas adoptadas por el nuevo presidente, se planteara, aunque más no sea en términos discursivos, la necesidad de acompañar estas medidas. Sin embargo, dado que, los posicionamientos políticos son relacionales en función de los intereses y capitales en disputa, Angeloz comenzó a esbozar algunas diferencias discursivas con Menem. Para el cordobés era necesario generar un marco de acuerdos con las diferentes fuerzas políticas y sociales que posibilitara la transformación de las relaciones sociales, el papel del Estado y el modelo económico (un ejemplo de ello sería el Consejo Económico y Social). Sin embargo, la orientación general de las intervenciones estatales sobre el mercado de trabajo demostró una clara sintonía entre ambos gobiernos.

En primer lugar, las medidas adoptadas por el gobierno angelocista en materia económica, laboral y social se encuentran dentro del marco de los lineamientos que establecía la hegemonía neoliberal de aquellos años. En particular, el entonces gobernador destacaba en el mensaje a la Honorable asamblea legislativa en 1991 su “convencimiento de que hemos de seguir el rumbo de la apertura económica, de la activa inserción regional y mundial, de la reforma del sistema productivo en base a devolver protagonismo, competencia y libertad, a la sociedad civil, el rumbo en definitiva de las impostergables reformas que el país exige" (Angeloz, 1995, p. 285).

En segundo lugar, y en sintonía con lo planteado desde el gobierno nacional, bajo "la voluntad de la conducción política de transformación del sistema del área del trabajo que el país requiere" 20 el proceso de transformación del sistema de relaciones de trabajo, y las ideas que lo sustentaban, se asentaban en "la necesidad de promover una descentralización de los sistemas de negociación colectiva". ${ }^{21}$ Desde la mirada ministerial, por su parte, el sistema vigente, y único hasta el momento, de negociaciones colectivas respondía "a un proyecto político de concentración de poder, que por otra parte se ajustó a un modelo económico proteccionista y centrado, [que] se ha agotado en

\footnotetext{
${ }^{20}$ BHLC, IMT, 1991, p. 153.

${ }^{21}$ BHLC, IMT, 1991, p. 153.
} 
el país". 22 Esto significaba, por cierto, la descentralización de la negociación y la concertación colectiva "abierta", tanto para el ámbito privado como para el público.

Así, durante estos años además de la ejecución de labores de contralor y vigilancia de aplicación de las normas, la solución de conflictos colectivos e individuales y de asistencia al empleo, hubo una clara direccionalidad tendiente a la descentralización de la toma de decisiones que estructuraban y regulaban el trabajo. Un ejemplo de ello fue la consolidación y creación de nuevas Comisiones de Concertación de las Condiciones y Medio Ambiente de Trabajo tripartitas ${ }^{23}$ que, si en los primeros años abarcaban la totalidad de una actividad, hacia el final del período se negociaba y acordaba a nivel de empresas. Por otro lado, si a comienzos de 1991 el Ministerio de Trabajo sentenciaba que estaba impulsando fuertemente a nivel provincial y a través de sus representantes a nivel nacional, ${ }^{24}$ la descentralización de la negociación colectiva para el ámbito privado, ${ }^{25}$ en el ámbito laboral público comenzaron tempranamente las regulaciones de las relaciones laborales también desde esta perspectiva descentralizadora. ${ }^{26}$

Por otro lado, consideramos relevante destacar que desde 1990 las tareas del Ministerio de Trabajo se orientaron, además, a la elaboración de una propuesta que luego se elevará al ámbito nacional, sobre la habilitación de contratos de trabajo a tiempo parcial y el pago de contribuciones de seguridad social "sobre la base de la remuneración efectivamente percibida por el trabajador" ${ }^{27}$ Este proyecto de acuerdo con la visión del Ministerio se orientaba a reducir los costos laborales de los empresarios que se suponía abonaría a la creación de nuevos puestos de trabajo. Quisiéramos destacar la mencionada propuesta, que es anterior a la Ley de Empleo № 24.013 -sancionada el 13 de noviembre y promulgada el 5 de diciembre de 1991- que estableció las nuevas modalidades de contrato de trabajo flexible y jornadas reducidas de trabajo. ${ }^{28}$

Por otra parte, las actividades en materia de "reconversión productiva" se intensificaron a partir de 1993, ya que desde el Ministerio de Trabajo de la provincia se reconocía "la gravedad del problema de la desocupación en nuestro tiempo. Por ello se ha hecho necesario trabajar duramente en la creación de puestos de trabajo, y en la

\footnotetext{
22 BHLC, IMT, 1991, p. 153.

${ }^{23}$ BHLC, IMT, 1991; 1992; 1993.

${ }^{24}$ Como parte del proceso de descentralización del sistema de relaciones laborales, el Ministerio de Trabajo de la provincia de Córdoba promovió la creación (desde 1989 e institucionalizándose en 1990) del Consejo Federal de Administraciones Provinciales del Trabajo. BHLC, IMT, 1991, p. 156.

${ }^{25}$ BHLC, IMT, 1991, p. 155.

${ }^{26}$ Tempranamente, en 1989 a través del decreto 6537/89 se había creado la comisión de política laboral docente integrada por el Ministerio de Educación y la Unión de Educadores de la Provincia, así como la Comisión de relaciones laborales de la Salud; ambas tendientes a la fragmentación de las negociaciones y con consecuencias significativas para el conjunto de trabajadores estatales.

${ }^{27}$ BHLC, IMT, 1991, p. 160. Dicha propuesta había comenzado a bosquejarse a fines del año 1989.

${ }^{28}$ Estas modificaciones establecidas por el nuevo marco legal fueron condición sine qua non para el desenvolvimiento de la estrategia de acumulación vigente, que flexibilizó y precarizó las condiciones de trabajo y contratación (Cortés y Marshall, 1991).
} 
reinserción laboral del desocupado. Ello se ha alcanzado en base a programas de formación ocupacional y de colocaciones." 29

En relación a la promoción del empleo privado, el Departamento Servicio de Empleo profundizó en estos años la modificación de una concepción asistencial de intervención a otra que otorgaba centralidad a "la eficiencia, la productividad y la adaptación positiva de la fuerza de trabajo a los cambios estructurales, respondiendo a la efectiva demanda del mercado de trabajo", con una creciente focalización hacia los sectores que consideraban con mayor vulnerabilidad. ${ }^{30}$

Así, en un contexto de creciente desempleo, ${ }^{31}$ hacia 1993 se implementó un "Programa Provincial de Promoción del Empleo" para profundizar las mediciones de la situación del mercado laboral urbano en el interior provincial. Se entendía que esto posibilitaría realizar diagnósticos eficaces para la elaboración de políticas públicas de alto impacto en la población-objetivo. Por otro lado, se implementó un Subsistema de colocaciones o intermediación de mano de obra, también destinado al empleo privado. ${ }^{32} \mathrm{~A}$ modo de ejemplo, para evaluar su impacto, en 1992 a nivel provincial hubo 4.177 inscriptos, de los cuales fueron colocados $1.615,33$ es decir un 38,66\%. Mientras que en 1994 cuando la tasa de desocupación alcanzó al 9,6\% de la población económicamente activa de la provincia hubo 2.701 inscriptos de los cuales se colocaron apenas a 831. Asimismo, en el marco de propuestas para la reconversión y adaptación de la mano de obra a las nuevas condiciones del mercado de trabajo urbano y de la demanda de fuerza de trabajo, se implementó un nuevo "Programa Provincial de Formación Ocupacional", en conjunto con Nación, Municipios e instituciones privadas en el que se "capacitaba" a la mano de obra. ${ }^{34}$

\footnotetext{
${ }^{29}$ BHLC, IMT, 1994, p. 155.

${ }^{30}$ BHLC, IMT, 1994, p. 156.

${ }^{31} \mathrm{Si}$ bien desde el Ministerio de Trabajo se aseguraba que "la provincia de Córdoba es uno de los estados que exhibe una menor cuota de desempleo en el concierto nacional, pero ello no quiere decir que la cuestión no sea preocupante" (BHLC, IMT, 1995, p. 161), los datos estadísticos reconstruidos a partir de la Encuesta Permanente de Hogares del INDEC nos muestran que para 1995 la desocupación en Córdoba era más alta que la del total de aglomerados del interior del país.

${ }^{32}$ El sistema de colocaciones de mano de obra -en épocas anteriores conocido como Bolsa de Trabajo- tenía como objetivos "contribuir a la colocación individual, masiva y especializada de trabajadores y captar las oportunidades de empleo, procurando el adecuado ajuste ocupacional o profesional entre los demandantes y oferentes". BHLC, IMT, 1995, p. 165.

${ }^{33}$ Lamentablemente no contamos con información detallada acerca de las localidades en donde se desarrolló esta política pública ni tampoco las actividades y empresas en los que se "colocaron" estos trabajadores, lo que nos posibilitaría poder contar con un panorama más general al tiempo que incorporar nuevas variables y fuentes.

${ }^{34}$ En 1993 se inscribieron a los cursos brindados 2.500 trabajadores en la provincia, aunque sólo se egresaron 512 trabajadores (59 en construcción, 295 en servicios, 101 en la industria del vestido y 51 de la industria de la madera). BHLC, IMT, 1995, p. 162.
} 
Hacia el final de nuestro periodo se expresaba que, como parte de las denominadas políticas de descentralización activas para contribuir a la generación de nuevos empleos desde el Ministerio, se habían constituido en el interior de la provincia alrededor de veinte Juntas Regionales Promotoras de Empleo para el sector privado que funcionaban “como organismos participativos de promoción de empleo donde este Ministerio [de trabajo] conjuntamente con los medios de información y la intervención de los municipios y comunas" 35 realizaba acciones en ese sentido.

Asimismo, quisiéramos destacar que tempranamente para el sector público se estableció, en convenio con la UNC, un "Programa de Becas para Investigación y Capacitación de Carácter permanente" por medio del decreto 707 del 22/3/91, que también se encontraba en sintonía con lo que establecerá la Ley Nacional de Empleo. Estos sistemas de becas y pasantías desplazaron el acento de la asistencia a la capacitación ocupacional, orientada al desarrollo de habilidades compatibles con la polivalencia de funciones que demandaba ahora la administración pública. Los destinatarios (jóvenes estudiantes universitarios) eran considerados potencialmente "empleables". Así, desde la óptica del gobierno provincial, se argumentaba que los mismos se beneficiarían de estas políticas por la adquisición de ciertos saberes prácticos -desarrollo de condiciones y habilidades- que les daría la experiencia en las distintas áreas de la administración estatal. Es necesario remarcar que estas nuevas figuras legales posibilitaron la reducción, tanto en el ámbito público como privado, de los costos de contratación y del pago de salarios a través de estos sistemas de becas, constituyendo nuevas formas precarias de trabajo.

Respecto a las intervenciones del Ministerio de Trabajo en materia salarial, ${ }^{36}$ se celebraron diversos acuerdos de incrementos salariales que combinaban "criterios de productividad con eficiencia de la mano de obra y mayor producción" 37 con otros que no lo hacían. Asimismo, en sus funciones de policía del trabajo, el número de inspecciones generales entre 1990 y 1992 fue en descenso y en los años posteriores registraron leves ascensos interanuales sin alcanzar los niveles del punto de partida. Por su parte, las inspecciones de higiene y seguridad aumentaron considerablemente. Respecto a los arbitrajes y conciliaciones colectivas, fueron en descenso y, por el contrario, se incrementaron los individuales y comenzaron a aparecer casos de arbitrajes y conciliaciones plurindividuales. Esto podría indicar, por un lado, los cambios en la legislación laboral, en particular la Ley Nacional de Empleo y el Código procesal de trabajo de la provincia de Córdoba antes referido, y por otro, una modificación del repertorio de reclamos colectivos que antes encabezaban los gremios y sindicatos, ahora resueltos individualmente.

\footnotetext{
35 BHLC, IMT, 1995, p. 163.

36 Hasta el 18 de marzo de 1993, era necesario negociar el salario, teniendo en cuenta criterios de productividad, pero a partir de esa fecha el reemplazo del Decreto № 1334/91 del P.E.N. por el Decreto № 470/93 relativizó esta obligatoriedad.

${ }^{37}$ BHLC, IMT, 1994, p. 153.
} 
En el poder legislativo se iniciaron y trataron diversas medidas tendientes a la reregulación de las relaciones laborales del ámbito privado y se establecieron nuevas vinculaciones entre las jurisdicciones nacional y provincial respecto al mundo del trabajo. Así, entre 1991 y 1995 hubo una intensa modificación de las normativas laborales existentes tendiente a adaptarlas a las nuevas leyes nacionales, en donde, los acuerdos marco entre Nación y Provincia antes mencionados, también formaban parte de este proceso de simbiosis y complementariedad entre ambas. Esto, entre otros casos, lo podemos visualizar en el Convenio de asistencia y cooperación técnica y financiera entre el Ministerio de Trabajo de la Nación y de la Provincia de Córdoba. ${ }^{38}$ En la presentación para su aprobación, el diputado radical -y futuro ex sindicalista, ya que su actuación como diputado contribuyó de modo decisivo a su eclipse como dirigente- Harrington explicaba que el convenio se estableció “a fin de cubrir los baches producidos por la readecuación del Estado y el aparato productivo". ${ }^{39}$ En su exposición reconocía que

la formación profesional como claramente su concepción lo indica, está compuesta de aspectos esenciales sin los cuales sería imposible su concreción, como es el caso de la información como medio de conocer la realidad laboral y por ende los requerimientos de la oferta y la demanda, y sobre estas bases surge la capacitación como otro aspecto de importancia a través de los cuales se asienta el sentido estricto del convenio desembocante en el rubro más importante que es la reinserción ocupacional. ${ }^{40}$

Podemos reconocer en esta afirmación la consideración (sin oposición por parte de los otros bloques legislativos) de que las herramientas que desde el Estado podían brindarse a los trabajadores (desocupados o no), en términos de información, los colocaría en una situación de "igualdad de elección" en el mercado laboral, para "reinsertarse" o permanecer en él. Nuevamente aparece el supuesto de que la situación de desempleo es una problemática de tipo individual, plausible de ser modificada con herramientas (in)formativas. Más específicamente, se asume que es objetivo del mencionado convenio "adecuar la demanda de formación con la demanda ocupacional, calificar y convertir la mano de obra con problemas de inserción" ${ }^{41}$, etcétera.

En este contexto se propusieron otras intervenciones estatales como el Programa de capacitación laboral "Proyecto Joven”, Fase I, que entró en ejecución en 1994 e intentaba "mejorar las posibilidades de inserción laboral de los beneficiarios, brindándole

\footnotetext{
${ }^{38}$ Dicho convenio, respondía a la adecuación del inciso "e" del artículo 2 de la Ley Nacional de Empleo (24.013), que establecía la necesidad de "Incorporar la formación profesional como componente básico de las políticas y programas de empleo".

${ }^{39}$ BHLC, DSD, $1994(8 / 6)$, p. 617.

${ }^{40}$ BHLC, DSD, 1994 (8/6), p. 617.

${ }^{41}$ BHLC, DSD, 1994 (8/6), p. 617.
} 
capacitación intensiva e integral en ocupaciones semi-calificadas que demandaba el sector productivo y la oportunidad de realizar una práctica laboral en el ámbito de la empresa" (Andalle, 1999). Los cursos tenían un carácter intensivo y práctico de cuatro meses y requerían como contraprestación del dinero recibido, su realización. Estas intervenciones destinadas a los jóvenes pueden ser aglutinadas bajo los preceptos antes expuestos. ${ }^{42}$

Por otra parte, destacamos otras propuestas legislativas cuyo objetivo fue crear políticas de empleo activas para los considerados "inempleables" fomentando la incorporación de trabajadores en ámbitos laborales diversos o la creación de cooperativas apoyadas por el Estado que tenían la misma finalidad de "reinsertar" socialmente a través de su incorporación al mercado de trabajo. ${ }^{43}$ Tal como plantea Campos Ríos (2003), este tipo de políticas restringidas fueron las primeras en utilizar el concepto de empleabilidad para el acceso a un puesto de trabajo. En el caso de nuestra provincia nuevamente como modus operandi, la primera medida que se adoptó fue el registro, ${ }^{44}$ la realización de un diagnóstico y luego la generación de propuestas tendientes a modificar o intervenir sobre la situación tematizada.

Otra línea de intervención social del estado que podríamos caracterizar importante en estos años para el ámbito privado por parte del Poder Legislativo es la referida a los diversos programas de Formación, Capacitación y creación de Regímenes de Pasantías, en consonancia con el Poder Ejecutivo (provincial y nacional). Estos programas en sus formulaciones presentan algunos elementos que pueden dar cuenta de las calificaciones requeridas y valoradas en el mercado de trabajo según la visión propia de los representantes del momento, así como de la aceptación de las teorías del capital humano para analizar el mercado de trabajo local. Es necesario considerar que, a partir de los años ochenta, comenzó un cuestionamiento en torno a la posibilidad de generar una sociedad y formas económicas de producción social con empleo tanto público como privado para toda la población activa, al tiempo que se profundizaron las transformaciones de la organización del trabajo, tornando más "flexibles" las actividades y decisiones de cada trabajador en el ámbito laboral. En este contexto, los requerimientos

\footnotetext{
${ }^{42}$ En las propuestas presentadas ante la Honorable Asamblea Legislativa encontramos, además, un intento por parte del Estado provincial de diagnosticar la situación referente a los jóvenes, a partir de la "Creación de registro de promoción de capacitación laboral de los jóvenes. Departamento Provincial de Trabajo", propuesta presentada en la sesión del 16 de septiembre de 1992 por los diputados Rodríguez Machado (UDC) y Correa (PJ). BHLC, DSD, 1992 (16/9), p. 1881. En relación a los posicionamientos que argüían la importancia de estas medidas como forma de dotar de capacidades (teóricas y prácticas) a estos jóvenes, de manera de "prepararlos para el mundo del trabajo", colocando el énfasis en la empleabilidad como requisito previo a cualquier inserción laboral.

${ }^{43}$ Proyecto presentado por el diputado Dip. Deheza de la Confederación Federal Independiente. BHLC, DSD, 1994 (31/8), p. 1294. En el mismo sentido, se puede entender la propuesta de "Declaración de la promoción de inserción laboral de disfuncionales", presentada por el Diputado radical Pereyra. BHLC, DSD, 1994 (21/9), p. 1519.

${ }^{44}$ Creación del registro judicial de incapacidad laboral, BHLC, DSD, 1994 (13/4; 18/5), pp. 129, 462-464; Censo y determinación de inserción laboral de personas disfuncionales en el ámbito provincial, BHLC, DSD, 1994 (21/9), p. 1519.
} 
del capital sobre las características y el volumen necesario de trabajadores a emplear también van a verse modificados.

Cabe destacar, además, que, bajo una concepción neoclásica del mercado de trabajo, que pareciera dominar en el discurso político cordobés del momento, el concepto de empleabilidad se asocia a una concepción única y unívoca (homogénea) del mercado laboral. Según esta concepción, al igual que en el funcionamiento de otros mercados, el de trabajo, se basa en un supuesto de competencia perfecta y en la libertad de elección de individuos que gozan de información plena para decidir sus acciones de modo de maximizar sus beneficios y minimizar sus pérdidas. Bajo estos lineamientos, la noción de empleabilidad es concebida como la capacidad de los sujetos de formar parte de esta relación de intercambio (Spinosa, 2005, p. 5). El Estado se limitaría a intervenir, entonces, para garantizar el acceso a la información o dotar de esas capacidades (por caso educación-formación) a aquellos individuos no las poseen, tanto en el ámbito público como privado. Asimismo, al considerarse el mercado de trabajo como instancia independiente y homogénea, se supone que "son los sujetos que pretenden participar los que deben modificar sus condiciones para acceder a él” (Spinosa, 2005, p. 13).

\section{Notas finales}

Nuestra argumentación intentó dar cuenta que la provincia de Córdoba sintió de manera aguda las reestructuraciones económicas desde temprano, producto del papel que había ocupado nuestra provincia en el anterior régimen social de acumulación. Asimismo, pudimos exponer que, pese a que discursivamente el gobernador de Córdoba planteaba distanciamientos y diferenciaciones con el gobierno nacional, en términos de las intervenciones sociales del estado orientadas al empleo tanto público como privado, por el contrario, encontramos numerosos puntos de confluencia que quedaron plasmados en acuerdos, programas y en una profusa readecuación legislativa en materia laboral con una orientación claramente neoliberal.

Como hemos podido analizar a lo largo del artículo, a partir de las intervenciones orientadas hacia el mercado de trabajo urbano, podemos dividir dos grandes momentos. Uno primero que podríamos denominar de normalización institucional, en donde debemos resaltar la Creación de la Secretaría Ministerio de Trabajo y el acta acuerdo firmada entre el gobierno provincial y el nacional en donde se redelimitaron las funciones entre una y otra jurisdicción. Asimismo, esta primera etapa estuvo dedicada a la restitución en el interior provincial de subdelegaciones e inspectorías del Ministerio de Trabajo de la Nación, así como a la restitución de los puestos de trabajo a quienes arbitrariamente habían sido desplazados por la última dictadura cívico-militar. Si bien estas intervenciones sociales del estado tuvieron como eje el ámbito público, hubo también intervenciones destinadas al ámbito privado. 
El segundo momento que periodizamos, entre la aprobación de la nueva Constitución provincial en 1987 y la salida anticipada de Angeloz en 1995, está atravesado por un acontecimiento de relevancia para nuestro objeto de estudio como es la aprobación de la Ley Nacional de Empleo. Pese a las particularidades de cada año, pudimos identificar las tempranas discusiones en torno a la necesidad de modificar las regulaciones del mercado de trabajo y las propuestas de reorientación de la fuerza de trabajo de acuerdo a los nuevos requerimientos del sistema productivo. El resultado de estas tematizaciones fue una re-regulación de las relaciones laborales, que se plasmó en modificaciones legislativas del período que comenzó en 1991, con la sanción de la nueva Ley Nacional de Empleo, y obligó a un proceso de readecuación de las normativas laborales en el ámbito provincial, gracias a la creciente descentralización estatal que otorgaba mayores capacidades al Estado provincial para la regulación del trabajo. Así, al momento de realizarse estas modificaciones, cristalizaron los debates y acuerdos que habían comenzado a desarrollarse años atrás, promovidas por actores locales.

A partir de lo expuesto, podemos afirmar que, si bien la bibliografía existente plantea que el predominio provincial del partido radical marcó un proceso distintivo en relación al ámbito nacional, fundamentalmente a partir de 1989, y que dio como resultado un "modelo cordobés" con rasgos bienestaristas, nuestro análisis permite relativizar o matizar estas lecturas. Sabiendo incompletas estas páginas, esperamos haber iniciado un camino que aporte a complejizar el estudio de la historia reciente de Córdoba y, más general, las características de las transformaciones económicas producidas a partir de la recuperación democrática en Argentina en clave provincial.

\section{Referencias bibliográficas:}

Almada, J. y Reche, F. (2019). La economía cordobesa en el marco de la última dictadura cívico-militar: cambios en los albores de la valorización financiera (Argentina, 19761983). Coordenadas VI (1), 92-111.

Almada, J., Giraudo, M. y Schuster, E. (2015). El trabajo en Córdoba. Una mirada de largo plazo. En C. La Serna (Comp.), Estado, política pública y acción colectiva: praxis emergentes y debates necesarios en la construcción de la democracia, (pp. 402-413) Córdoba, Argentina: UNC. [E-book: http://blogs.unc.edu.ar/doctoradoaypp/files/ebookcoloquio-iifap-final.pdf] (descarga 13/4/16).

Andalle, L. (1999). El desempleo de jóvenes en la ciudad de Córdoba y políticas públicas de empleo (Tesis de Maestría). IIFAP, Universidad Nacional de Córdoba, Córdoba.

Angeloz, E. (1995). La aventura de la democracia, 12 años de realizaciones. Mensajes ante las Honorables Asambleas Legislativas. Córdoba: Ministerio de Coordinación de Córdoba.

Basualdo, E. (2010). Estudios de Historia Económica Argentina desde mediados del siglo XX a la actualidad. Buenos Aires: Siglo XXI.

Bil, D. A. (2017). La crisis mundial del sector automotriz (1978-1982) y los efectos sobre el complejo en Argentina. Projeto História: Revista do Programa de Estudos Pós-Graduados de História 60, 281-318. 
Brennan, J. y Gordillo, M. (2008). Córdoba rebelde. El Cordobazo, el clasismo y la movilización social. La Plata: De la campana.

Campos Rios, G. (2003). Implicaciones económicas del concepto de empleabilidad. Aportes 23 (VII), 101-111.

Carrizo, C. (1997). Intervención estatal y organizaciones sociales. El consejo económico y social de la Provincia de Córdoba. 1985-1993. Administración pública y sociedad 10, 122-147.

Closa, G. (2010). La recuperación de la democracia y los gobiernos radicales. Angeloz y Mestre (1983-1999). En C. Tcach (Coord.), Córdoba Bicentenaria: claves de su historia contemporánea. (pp. 481-510) Córdoba: CEA - Editorial de la UNC.

Cortés, R. y Marshall, A. (1991). Estrategias económicas, intervención social del Estado y regulación de la fuerza de trabajo. Argentina 1890-1990. Estudios del Trabajo 1, 21-46.

Cortés, R. y Marshall, A. (1993). Política social y regulación de la fuerza de trabajo. Cuadernos médico-sociales 65/66, 3-12.

Fantín, I. y Schuster, E. (2013). El IERAL y las reformas estructurales del Estado en Córdoba. En S. Morón y S. Roitman (Eds.), Procesos de acumulación y conflicto social en la Argentina contemporánea: debates teóricos y estudios empíricos. (pp. 81-100) Córdoba: Universitas.

Fitzsimons, A. (2013). Producción, relaciones sociales y valor: una crítica a la teoría del patrón de acumulación basado en la valorización financiera. Razón y Revolución 24, 85-103.

Fernández, A. (Comp.) (2005). Estado y relaciones laborales: transformaciones y perspectivas. Buenos Aires: Prometeo.

Gatto, F., Gutman, G. y Yoguel, G. (1988). Reestructuración industrial en la Argentina y sus efectos regionales: 1973-1984. Documento de trabajo, 14. Buenos Aires: CFI - CEPAL.

Gaudio, R., y Tomada, C. (1991). El restablecimiento de la negociación colectiva en Argentina (1988-1989). Boletín Informativo Techint (267), 98-121.

Gerchunoff, P. y Llach, L. (1999). El ciclo de la ilusión y el desencanto. Un siglo de políticas económicas argentinas. Buenos Aires: Ariel.

Gordillo, M. (2003). El modelo cordobés y su crisis: la caída de Angeloz en 1995. Anuario de la Escuela de Historia 3(3), 249-269.

Grigera, J. (2008). La desindustrialización y la formación de la clase obrera en Argentina (1976-2001). En V Jornadas de Sociología de la UNLP. Universidad Nacional de La Plata. Facultad de Humanidades y Ciencias de la Educación. Departamento de Sociología, La Plata.

Grigera, J. (2013). Esperando a EP Thompson. Desindustrialización y formación de clases sociales en Argentina (1976-2001). Mundos do Trabalho 5(10), 71-88. 
James, D. (1981). Racionalización y respuesta de la clase obrera: contexto y limitaciones de la actividad gremial en la Argentina. Desarrollo Económico 83 (21), 321-349.

Kabat, M. et al. (2014). Avances y retrocesos de la flexibilidad laboral en la Argentina. Aportes para una comparación de las trayectorias históricas de distintas ramas de actividad. Revista Mundo do trabalho 12 (6), 273-297.

La Serna, C. (1997). Actores y procesos en la crisis del estado de bienestar. Administración pública y sociedad (10), 101-121.

La Serna, C. (2001). La Democracia como Límite: el proceso de reforma del estado de la provincia de Córdoba en el Período 1995/1999. Ponencia presentada en el 1er. Congreso Argentino de Administración Pública. Rosario: Asociación de Administradores Gubernamentales..

Lindenboim, J. y González, M. (2004). El neoliberalismo al rojo vivo: el mercado de trabajo en Argentina. En E. Laurelli (Comp.), Nuevas territorialidades: desafíos para América Latina frente al siglo XXI. (pp. 31-50) La Plata: Ediciones Al Margen CESLA.

Marshall, A. (1996). Protección del empleo en América Latina: las reformas de los años 1990 y sus efectos sobre el mercado de trabajo. Revista Estudios del Trabajo (11), 3-28.

Marshall, A. (2000). Efectos sociales y económicos de la legislación del trabajo: debates y evidencias. En E. de la Garza (Coord.), Tratado Latinoamericano de Sociología del Trabajo. (pp. 451-470) México: El Colegio de México, Universidad Autónoma Metropolitana, Fondo de Cultura Económica.

Marticorena, C. (2010). Contenidos de la negociación colectiva durante la década de 1990 y la posconvertibilidad en actividades seleccionadas de la industria manufacturera. Estudios del Trabajo 39/40, 59-87.

Meik, M. y Zas, O. (1990). Desregulación y flexibilización normativa de la protección en el ordenamiento laboral argentino. En P. Galin y M. Novick (Comp.), La precarización del empleo en la Argentina (pp. 47-85) Buenos Aires: CEAL.

Neffa, J. C. (1998). Modos de regulación, regímenes de acumulación y sus crisis en Argentina (1880-1996). Buenos Aires: EUDEBA.

Novaro, M. y Palermo, V. (2003). La dictadura militar, 1976-1983: del golpe de estado a la restauración democrática. Buenos Aires: Paidós.

Ortiz, R. y Schorr, M. (2006). La economía política del gobierno de Alfonsín: creciente subordinación al poder económico durante la década perdida. En A. Pucciarelli (Ed.), Los años de Alfonsín: ¿El poder de la democracia o la democracia del poder? (pp. 291-333). Buenos Aires: Siglo XXI.

Palermo, V. y Novaro, M. (Comps.). (2004). La historia reciente. Argentina en democracia. Buenos Aires: Edhasa.

Palomino, H. (2005). Los cambios en el mundo del trabajo y los dilemas sociales. En J. Suriano (Dir.), Dictadura y democracia (1976-2001). Colección Nueva Historia Argentina, T. X. (pp. 377-390) Buenos Aires: Sudamericana. 
Peñalva, S. (2001). Regulaciones económicas y (des)protección social en la sociedad salarial. Las reformas orientadas al mercado y sus implicaciones institucionales en la Argentina. Ciclos en la historia, la economía y la sociedad (21), 181-223.

Pérez Álvarez, G. (2013). Una discusión con el concepto de “desindustrialización” desde el caso del noreste de Chubut. Revista de Estudios Marítimos y Sociales 5/6, 177-185.

Pérez, P. (2005). Los sospechosos de siempre. Los desocupados de larga duración en Argentina y su (in)empleabilidad. En J. C. Neffa (et al.), Desequilibrios en el Mercado de trabajo argentino. Los desafíos de la posconvertibilidad. (pp. 33-50) Buenos Aires: Ceil Piette Conicet.

Reche, F. (2015). Debates intelectuales en la historia económica argentina: una historia de la tesis del agotamiento del Modelo de Industrialización por Sustitución de Importaciones (Tesis de Licenciatura en Historia). Escuela de Historia (FFyH-UNC), Córdoba.

Rofman, A. y Romero, J. (1997). Sistema socioeconómico y estructura regional en la Argentina. Buenos Aires: Amorrortu Editores.

Servetto, A. y Moyano, J. (2009). Claves para la investigación de la historia política en los espacios locales y regionales. Estudios 18 (22), 9-18.

Sartelli, E., Kabat, M. et. al. (1998). Taylorismo, fordismo y post-fordismo en la Argentina: ¿la consolidación del régimen de gran industria? Razón y Revolución 4, 111.

Spinosa, M. (2005). Del empleo a la empleabilidad, de la educación a la educabilidad. Mutaciones conceptuales e individualización de los conflictos sociales. Ponencia presentada en el $7^{0}$ Congreso Nacional de Especialistas de Estudios del Trabajo. Buenos Aires: ASET.

Tarditi, R. (1999). Cambios en el proceso de producción en la rama automotriz a escala mundial. Notas introductorias para su descripción. Comunicación de Investigación del Programa de Investigación sobre el Movimiento de la Sociedad Argentina, pp. 1-5.

Tarditi, R. (2000). La crisis mundial de los' 70 y los' 80 observada a partir de la evolución de la producción automotriz. Documento de Trabajo 23, 1-29.

Zorzoli, L. (2015). La normativa sindical entre la dictadura y el alfonsinismo, propuesta de sistematización. En A. Schneider y P. Ghigliani (Comp.), Clase obrera, sindicatos y Estado. Argentina (1955-2010) (pp.147-169.) Buenos Aires: Imago Mundi.

Para citar este artículo:

Almada Julieta A. (2019). Estado, economía y política: las intervenciones sociales del estado orientadas al mercado de trabajo en la provincia de Córdoba entre 1983 y 1995. Anuario de la Escuela de Historia Virtual 15, 68-90. 